\title{
Dual effects of noradrenaline on astroglial production of chemokines and pro-inflammatory mediators
}

\author{
Ara E Hinojosa', Javier R Caso², Borja García-Bueno ${ }^{1}$, Juan C Leza ${ }^{1}$ and José LM Madrigal ${ }^{1 *}$
}

\begin{abstract}
Background: Noradrenaline (NA) is known to limit neuroinflammation. However, the previously described induction by NA of a chemokine involved in the progression of immune/inflammatory processes, such as chemokine (C-C motif) ligand 2 (CCL2)/monocyte chemotactic protein-1 (MCP-1), apparently contradicts NA anti-inflammatory actions. In the current study we analyzed NA regulation of astroglial chemokine (C-X3-C motif) ligand 1 (CX3CL1), also known as fractalkine, another chemokine to which both neuroprotective and neurodegenerative actions have been attributed. In addition, NA effects on other chemokines and pro-inflammatory mediators were also analyzed.
\end{abstract}

Methods: Primary astrocyte-enriched cultures were obtained from neonatal Wistar rats. These cells were incubated for different time durations with combinations of NA and lipopolysaccharide (LPS). The expression and synthesis of different proteins was measured by RT-PCR and enzyme-linked immunosorbent assay (ELISA) or enzyme immunoassays. Data were analyzed by one-way analysis of variance (ANOVA), followed by Newman-Keuls multiple comparison tests.

Results: The data presented here show that in control conditions, NA induces the production of CX3CL1 in rat cultured astrocytes, but in the presence of an inflammatory stimulus, such as LPS, NA has the opposite effect inhibiting CX3CL1 production. This inversion of NA effect was also observed for MCP-1. Based on the observation of this dual action, NA regulation of different chemokines and pro-inflammatory cytokines was also analyzed, observing that in most cases NA exerts an inhibitory effect in the presence of LPS. One characteristic exception was the induction of cyclooxygenase-2 (COX-2), where a summative effect was detected for both LPS and NA.

Conclusion: These data suggest that NA effects on astrocytes can adapt to the presence of an inflammatory agent reducing the production of certain cytokines, while in basal conditions NA may have the opposite effect and help to maintain moderate levels of these cytokines.

Keywords: Fractalkine, CX3CL1, MCP-1, CCL2, Astrocytes, Inflammation

\section{Background}

Noradrenaline (NA) is recognized as a key modulator in the regulation of multiple central nervous system (CNS) activities, such as mood changes [1], memory formation [2], stress response [3] and cellular energy metabolism [4], among others. However, in relation to the study of mechanisms involved in the development of diseases with a neurological component, the main attribute of

\footnotetext{
* Correspondence: jlmmadrigal@med.ucm.es

'Departamento de Farmacología, Facultad de Medicina, Universidad Complutense de Madrid (UCM), Centro de Investigación Biomédica en Red de Salud Mental (CIBERSAM), Instituto de Investigación Hospital 12 de Octubre (Imas12), Instituto de Investigación en Neuroquímica UCM, Avenida Complutense s/n, Madrid 28040, Spain

Full list of author information is available at the end of the article
}

NA is its ability to reduce the neuroinflammatory processes associated to them [5]. The degeneration of the main noradrenergic area in the brain, the locus coeruleus, seems to be one of the stages preceding the subsequent development of neuronal death observed in Alzheimer's disease [6]. In fact, diverse experimental settings indicate that NA interactions with different types of brain cells lead to the regulation of inflammatory pathways and mediators [5].

Based on this, a sound hypothesis proposes that the loss of NA constitutive levels can create a 'permissive' environment for the development of inflammation and subsequent neurodegeneration [7]. 
Among other roles, astrocytes are in charge of the surveillance of the CNS status, providing alarm signals when threat is detected and helping to maintain CNS homeostasis [8]. Thus, we have focused our work on the analysis of NA interaction with astrocytes, to help elucidate the means through which NA protects neurons against different types of injuries.

We previously observed that NA, through the activation of $\beta 2$-adrenergic receptors and the elevation of cAMP, induces the production of the chemokine $(\mathrm{C}-\mathrm{C}$ motif) ligand 2 (CCL2)/monocyte chemotactic protein 1 (MCP-1) and protects neurons against excitotoxicity [9]. This fact, while contradictory with the well-known actions of CCL2 as a chemoattractant that facilitate the progression of the immune and inflammatory responses (which can have fatal consequences for nearby cells) [10], is in agreement with several studies which describe neuroprotective actions of CCL2 against multiple types of injuries [11]. This observation led us to the analysis of NA regulation of different chemokines on astrocytes.

As their name suggests, all chemokines can attract those cells expressing the specific receptors. This explains their involvement in very disparate processes where cell migration is present, such as cell adhesion/ trafficking [12], angiogenesis [13] or progenitor cell migration [14]. In addition, as mentioned, chemokines by themselves may also cause some changes in cell functioning by direct interaction with such cells. In fact, new actions unrelated to the regulation of cell migration have been recently discovered for chemokines, highlighting the potential relevance of this family of proteins, particularly in the field of neuroinflammation [11].

One of the chemokines that has proven to play a significant role in the regulation of brain physiology is chemokine (C-X3-C motif) ligand 1 (CX3CL1), also known as fractalkine or neurotactin. CX3CL1 is expressed by neurons, astrocytes and microglia, and its specific receptor CX3CR1 is also expressed by all these cell types [15]. However, since the main production of CX3CL1 is observed in neurons and the receptor seems to be more abundant in glial cells, it has been proposed that CX3CL1 serves as an intermediary used by neurons to communicate with glial cells. While several studies have shown that CX3CL1 can modulate neuronal activity and survival, others indicate that the restrain of CX3CL1 activity can also prevent neuronal damage in certain pathologies [16-18]. This potential dual role of CX3CL1 has been proposed to be dependent on the different stages of certain neurodegenerative diseases where microglia activation may be beneficial (by their ability to remove apoptotic cells and toxic debris) or detrimental for neurons (by the elimination of healthy cells) [19].

The present study describes the induction of CX3CL1 expression and synthesis by NA in astrocytes. The results obtained also indicate that in the presence of a pro-inflammatory stimulus, such as lipopolysaccharide (LPS) from gram-negative bacteria, which causes a large production of CX3CL1 by astrocytes, NA has the opposite effect inhibiting CX3CL1 production. This observation led us to analyze if this also applies to CCL2 and other related chemokines, as well as different proinflammatory mediators. The data show that while each one of the proteins evaluated has a different regulation by NA, in most cases where NA induced their expression in control conditions, the presence of LPS switched NA effect towards inhibition. This suggests that, in the brain, NA may be responsible for the maintenance of the constitutive levels of certain factors, while it can repress the overproduction in inflammatory situations.

\section{Methods}

\section{Reagents}

Fetal calf serum $(<10 \mathrm{EU} / \mathrm{ml})$ and Dulbecco's modified Eagle's medium (DMEM) were obtained from Gibco Life Technologies (Carlsbad, CA, USA). LPS from Escherichia coli 0111:B4 and NA for cell treatments, and glutamine, penicillin and streptomycin for cell cultures, were obtained from Sigma-Aldrich (St Louis, MO, USA). TRIzol, Taq polymerase and cDNA synthesis reagents were obtained from Invitrogen (Carlsbad, CA, USA).

\section{Astrocyte cultures}

All experimental protocols adhered to the guidelines of the Animal Welfare Committee of the Universidad Complutense of Madrid, Spain, and according to European Union laws. Rat cortical astrocytes were obtained as previously described [20]. Briefly, 1-day-old Wistar rats (Harlan, Indianapolis, IN, USA) were used to prepare primary mixed glial cultures. Microglia were detached by gentle shaking after 11 to 13 days in culture. Astrocytes were prepared by mild trypsinization of the remaining cells, replated at $6 \times 10^{5}$ cells $/ \mathrm{ml}$, and consisted of $95 \%$ astrocytes as determined by staining for glial fibrillary acidic protein (GFAP) and $<5 \%$ microglial as determined by staining with the specific marker OX-42.

\section{mRNA analysis}

Total cytoplasmic RNA was prepared from cells using TRIzol reagent and aliquots were converted to cDNA using random hexamer primers. Quantitative changes in mRNA levels were estimated by real-time PCR (qPCR) using the following cycling conditions: 35 cycles of denaturation at $95^{\circ} \mathrm{C}$ for 10 seconds, annealing at 58 to $61^{\circ} \mathrm{C}$ for 15 seconds, depending on the specific set of primers, and extension at $72^{\circ} \mathrm{C}$ for 20 seconds. Reactions were carried out in the presence of SYBR green (1:10,000 dilution of stock solution from Molecular Probes, Eugene, OR, USA) and in a $20 \mu \mathrm{l}$ reaction in Rotor-Gene (Corbett 
Table 1 Primers used for RT-PCR

\begin{tabular}{lll}
\hline $\begin{array}{l}\text { Gene } \\
\text { name }\end{array}$ & Forward primer & Reverse primer \\
\hline CX3CL1 & 5'-AATCCCAGTGACCTTGCTCATCCA & 5'-TGGACCCATTTCTCCTTGGGTCA \\
CCL2 & 5'-TGCTGTCTCAGCCAGATGCAGTTA & 5'-TACAGCTTCTITGGACACCTGCT \\
CCL6 & 5'-TGTTCCAGCAGGGCATCTTCTCT & 5'-GCCTCATTTGCATGGAGAGCCATT \\
CCL7 & 5'-GGACCAATTCATCCACTTGCTGCT & 5'-TCTGATGGGCTTCAGCACAGACTT \\
CCL12 & 5'-TGAGTCCTCCAGCTCTCATTCCAA & 5'-TGAACACTGAATCTGGTCCAGCCA \\
CXCL16 & 5'-TGTCGCTGGAAGTGCTACTGTGA & 5'-TCTTGGACTGCAACTGGAACCTGA \\
IL-13 & 5'-ACCTGCTAGTGTGTGATGTTCCCA & 5'-AGGTGGAGAGCTTCAGCTCACAT \\
TNFa & 5'-CTGGCCAATGGCATGGATCTCAAA & 5'-AGCCTTGTCCCTTGAAGAGAACCT \\
IFNY & 5'-AAAGACAACCAGGCCATCAGCAAC & 5'-TCTGTGGGTTGTCACCTCGAACT \\
COX-2 & 5'-GCATTCTTGCCCAGCACTTCACT & 5'-TTAAAGTCCACTCCATGGCCCAGT \\
GAPDH & 5'-TGCACCACCAACTGCTTAGC & 5'-GGCATGGACTGTGGTCATGAG \\
\hline
\end{tabular}

CCL12 chemokine (C-C motif) ligand 12, CCL2 chemokine (C-C motif) ligand 2, CCL6 chemokine (C-C motif) ligand 6, CCL7 chemokine (C-C motif) ligand 7, COX-2 cyclooxygenase-2, CX3CL1 chemokine (C-X3-C motif) ligand 1, CXCL16 chemokine (C-X-C motif) ligand 16, GAPDH glyceraldehyde 3-phosphate dehydrogenase, IFNY interferon gamma, IL-1 $\beta$ interleukin-1 beta, TNFa tumor necrosis factor alpha.

Research, Mortlake, Australia). Primers for the genes of interest were designed based on the rat sequences deposited in GenBank (Table 1). Relative mRNA concentrations were calculated from the take-off point of reactions using included software, and glyceraldehyde 3-phosphate dehydrogenase (GAPDH) levels used to normalize data.

\section{CCL2, CX3CL1, CCL6 and TNFa measurement}

Protein levels in the incubation medium were detected using specific enzyme-linked immunosorbent assay (ELISA), carried out according to the manufacturer's instructions: R\&D Systems Inc (Minneapolis, MN, USA) for CX3CL1; BD Biosciences (San Jose, CA, USA) for CCL2; CUSABIO (Wuhan, China) for chemokine (C-C motif) ligand 6 (CCL6); and RayBiotech (Atlanta, GA, USA) for tumor necrosis factor alpha (TNF $\alpha$ ). Briefly, the medium was collected from the astrocyte cultures and stored at $-80^{\circ} \mathrm{C}$ until the day of the assay (avoiding repeated freeze-thaw cycles). A standard curve was generated using the standards provided in the kits. The assay detection limits were: 31.3 to $2,000 \mathrm{pg} / \mathrm{ml}$ for CCL2; 195 to $12,500 \mathrm{pg} / \mathrm{ml}$ for CX3CL1; 0.156 to $10 \mathrm{ng} / \mathrm{m}$ for CCL6; and 25 to $20,000 \mathrm{pg} / \mathrm{ml}$ for TNFa.

\section{$\mathrm{PGE}_{2}$ measurement}

Prostaglandin $\mathrm{E}_{2}\left(\mathrm{PGE}_{2}\right)$ levels in the incubation medium were measured using a specific enzyme immunoassay (EIA), carried out according to the manufacturer's instructions (Cayman Chemical, Ann Arbor, MI, USA). Briefly, the medium was collected from the astrocyte cultures and stored at $-80^{\circ} \mathrm{C}$ until the day of the assay (avoiding repeated freeze-thaw cycles). A standard curve was generated using the rat $\mathrm{PGE}_{2}$ standard provided in the kit. The assay detection limit was $15 \mathrm{pg} / \mathrm{ml}$.

\section{Data analysis}

All experiments were undertaken at least in triplicate. Data were analyzed by one-way analysis of variance (ANOVA), followed by Newman-Keuls multiple comparison tests. $P$ values $<0.05$ were considered significant.

\section{Results}

\section{NA induces $\mathrm{CX} 3 \mathrm{CL} 1$ synthesis and release in astrocytes}

An ELISA assay was used to evaluate the production of CX3CL1 and its release from cultured astrocytes. Different concentrations of NA ( 1 to $50 \mu \mathrm{M}$ ) were added to the culture medium and the cells were incubated for 6 or 24 hours. Six hours of treatment did not yield significant changes in the concentration of CX3CL1. However, when the incubation period was extended to 24 hours, NA treatment caused an increase with significant differences with respect to the 24-hour control group for concentrations above $10 \mu \mathrm{M}$. The concentration of $50 \mu \mathrm{M}$ did not cause a production of CX3CL1 larger than that observed for 10
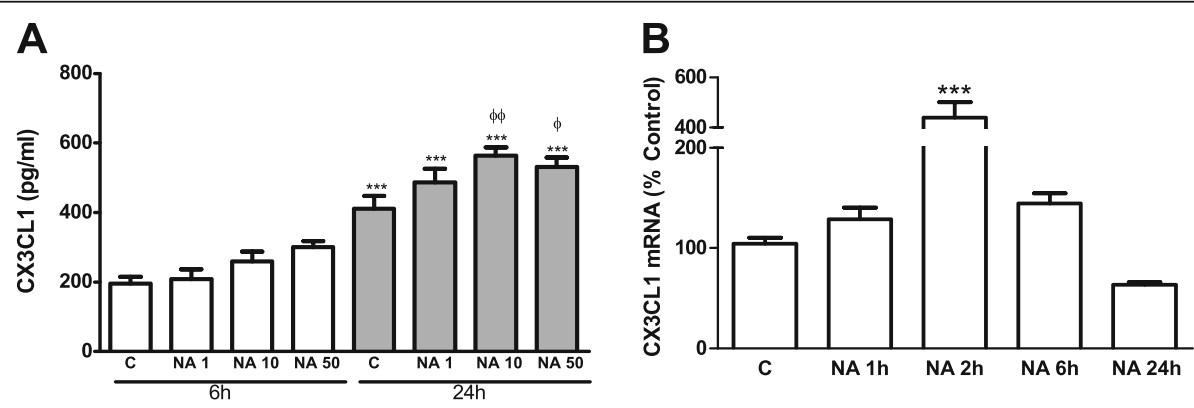

Figure 1 NA induces CX3CL1 production by astrocytes. (A) Astrocytes were incubated with control media or NA (1, 10 or $50 \mu \mathrm{M})$ for 6 or 24 hours. CX3CL1 levels in the media were assessed by ELISA. ${ }^{* *} P<0.001$ versus 6 -hour control; ${ }^{\Phi} P<0.05$ versus 24 -hour control; ${ }^{\Phi \Phi} P<0.01$ versus 24-hour control. Data are means \pm SE of $n=12$ replicates per group. (B) Astrocytes were incubated with control media or NA $10 \mu M$ for 1, 2, 6 or 24 hours. RNA was isolated and CX3CL1 mRNA levels determined by RT-PCR. Data are expressed as percentage of control values (set to 100\%). ${ }^{* * *} P<0.001$ versus control. Data are means $\pm \mathrm{SE}$ of $\mathrm{n}=8$ replicates per group. $\mathrm{C}$, control; CX3CL1, chemokine (C-X3-C motif) ligand 1; ELISA, enzyme-linked immunosorbent assay; NA, noradrenaline; RT-PCR, reverse transcription polymerase chain reaction; SE, standard error. 

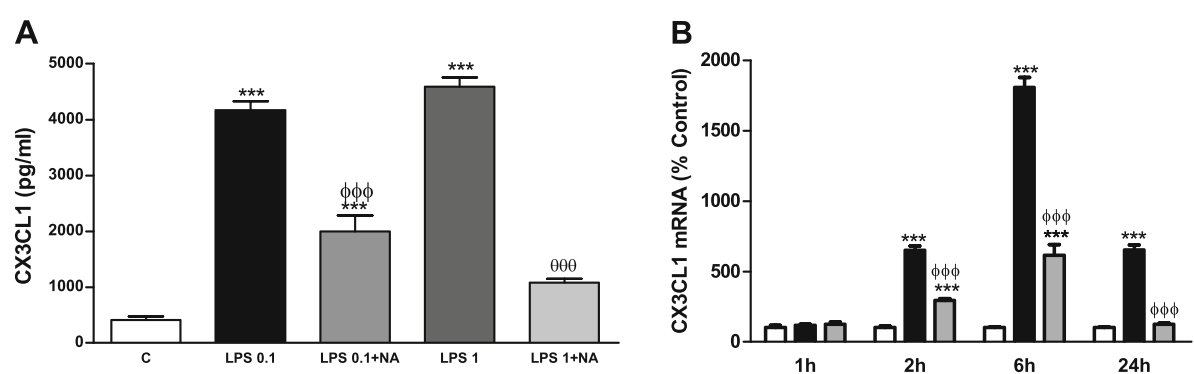

Figure 2 In the presence of LPS NA inhibits CX3CL1 production by astrocytes. (A) Astrocytes were incubated with control media, LPS 0.1 and $1 \mu \mathrm{g} / \mathrm{ml}$ alone or in combination with NA $10 \mu \mathrm{M}$ for 24 hours. CX3CL1 levels in the media were assessed by ELISA. ${ }^{* *} P<0.001$ versus control; ${ }^{\Phi \Phi \Phi} P$ $<0.001$ versus LPS $0.1 \mu \mathrm{g} / \mathrm{ml} ;{ }^{\theta \theta \theta} P<0.001$ versus LPS $1 \mu \mathrm{g} / \mathrm{ml}$. Data are means \pm SE of $\mathrm{n}=12$ replicates per group. (B) Astrocytes were incubated with control media (white columns), LPS $0.1 \mu \mathrm{g} / \mathrm{ml}$ (black columns) or LPS and NA $10 \mu \mathrm{M}$ (gray columns) for 1, 2, 6 or 24 hours. RNA was isolated and CX3CL1 mRNA levels determined by RT-PCR. Data are expressed as percentage of control values (set to 100\%). ${ }^{* *} P<0.001$ versus control; ${ }^{\Phi \Phi} P<0.001$ versus LPS. Data are means \pm SE of $n=8$ replicates per group. C, control; CX3CL1, chemokine (C-X3-C motif) ligand 1; ELISA, enzyme-linked immunosorbent assay; LPS, lipopolysaccharide; NA, noradrenaline; RT-PCR, reverse transcription polymerase chain reaction; SE, standard error.

$\mu \mathrm{M}$, suggesting that the amount measured represents NA maximal effect. Interestingly, the control values detected after 24 hours were higher than those detected after 6 hours (Figure 1A), confirming that CX3CL1 is constitutively released by astrocytes at considerable amounts.

Having found that $10 \mu \mathrm{M}$ is the lowest concentration of NA able to induce a significant induction of CX3CL1, we treated astrocytes for 1 to 24 hours with this amount of NA and used real-time RT-PCR (qRT-PCR) to assess mRNA levels of CX3CL1. This allowed us to observe an elevation that was maximal after 2 hours of incubation. Twenty-four hours after the onset of this treatment, the mRNA levels were lower than in the control group (Figure 1B).

\section{NA inhibits CX3CL1 production in the presence of LPS in astrocytes}

In order to evaluate the magnitude of NA effect, astrocytes were treated with an inflammatory stimulus known to induce CX3CL1 expression in the brain, such as LPS [21]. The incubation with LPS $0.1 \mu \mathrm{g} / \mathrm{ml}$ for 24 hours caused a greater than tenfold elevation of CX3CL1 levels in the culture media as assessed by ELISA (Figure 2A). This indicates that the elevation of CX3CL1 production caused by NA is only minor in relation to the full potential of these cells.

Having previously found the ability of NA to induce CX3CL1 by itself, we decided to analyze its possible interaction with LPS. We observed that it reduces the production of CX3CL1 caused by LPS (Figure 2A) even when a tenfold higher concentration of this endotoxin is used.

Parallel changes were found for CX3CL1 mRNA where LPS caused a large increase that was maximal after 6 hours of treatment and still remained elevated after 24 hours, and NA inhibited LPS effect on CX3CL1 mRNA reducing it to control levels after 24 hours of coincubation (Figure 2B).
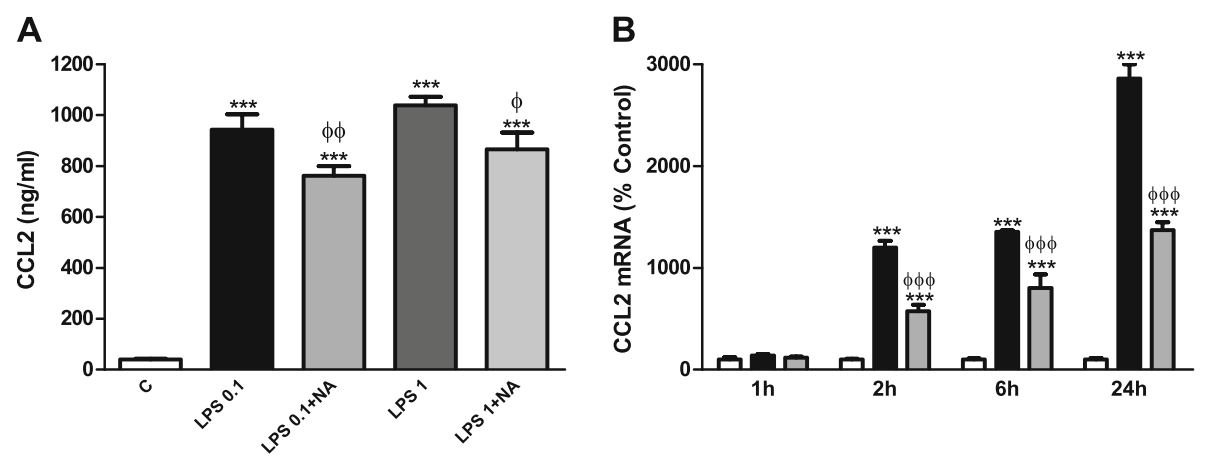

Figure 3 NA inhibits CCL2 production by astrocytes in the presence of LPS. (A) Astrocytes were incubated with control media, LPS 0.1 and $1 \mu \mathrm{g} / \mathrm{ml}$ alone or in combination with NA $10 \mu \mathrm{M}$ for 24 hours. CCL2 levels in the media were assessed by ELISA. ${ }^{* *} P<0.001$ versus control; ${ }^{\Phi} P$ $<0.05$ versus $L P S 1 \mu \mathrm{g} / \mathrm{ml}$; ${ }^{\Phi \Phi} P<0.01$ versus LPS $0.1 \mu \mathrm{g} / \mathrm{ml}$. Data are means \pm SE of $n=12$ replicates per group. (B) Astrocytes were incubated with control media (white columns), LPS $0.1 \mu \mathrm{g} / \mathrm{ml}$ (black columns) or LPS and NA $10 \mu \mathrm{M}$ (gray columns) for 1, 2, 6 or 24 hours. RNA was isolated and CCL2 mRNA levels determined by RT-PCR. Data are expressed as percentage of control values (set to 100\%). ${ }^{* * *} P<0.001$ versus control; ${ }^{\text {ФDథ } P}$ $<0.001$ versus LPS. Data are means \pm SE of $n=8$ replicates per group. C, control; CCL2, chemokine (C-C motif) ligand 2; ELISA, enzyme-linked immunosorbent assay; LPS, lipopolysaccharide; NA, noradrenaline; RT-PCR, reverse transcription polymerase chain reaction; SE, standard error. 


\section{NA inhibits CCL2 production in the presence of LPS in astrocytes}

Since NA presented this double effect for CX3CL1 expression, we tested if it would also have a similar effect for CCL2, another chemokine we previously found that can be induced by NA in astrocytes [9]. ELISA and qRT-PCR studies allowed us to detect a similar pattern of regulation, where LPS caused a large induction of CCL2 that was in part prevented by the co-treatment with NA (Figure 3).

\section{Alterations in NA regulation of different chemokines}

While each chemokine has particular features that differentiate it from the rest, they all have other features in common, and the dual regulation by NA could be one of the characteristics that apply to all of them. Due to the large number of chemokines known to date, a preliminary approach was made by analyzing mRNA regulation in three chemokines with effects similar to those of CCL2 and that share the C-C chemokine receptor type 2 (CCR2) with CCL2, such as CCL6/C10, chemokine (C-C motif) ligand 7 (CCL7)/monocyte chemotactic protein-3 (MCP-3) and chemokine (C-C motif) ligand 12 (CCL12)/monocyte chemotactic protein-5 (MCP-5) [22]. Chemokine (C-X-C motif) ligand 16 (CXCL16)/small inducible cytokine subfamily B member 16 (SCYB16) was also studied because it has also been characterized as a neuroprotective agent that modulates astroglial production of CCL2 [23].
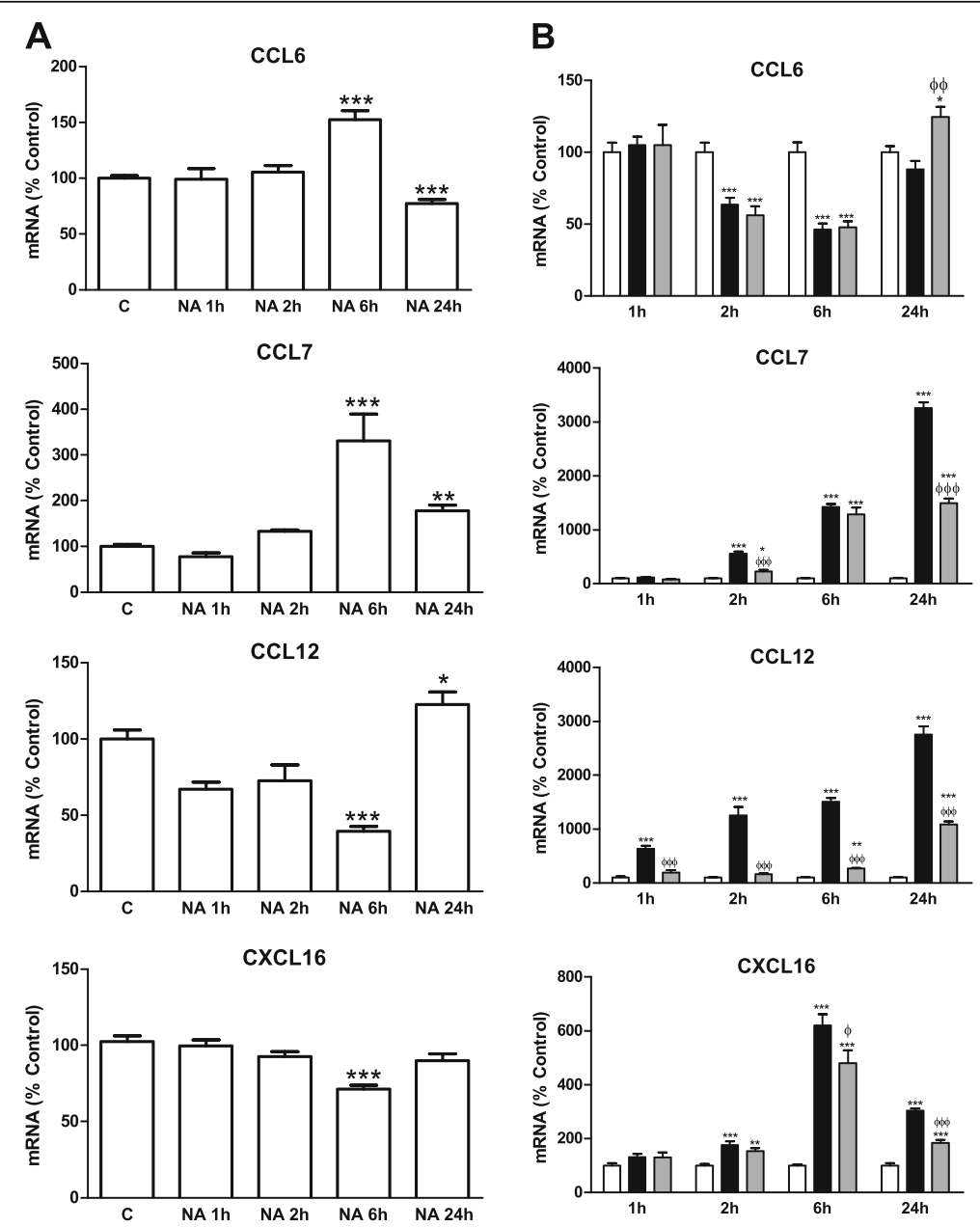

Figure 4 NA effects on different chemokines. (A) Astrocytes were incubated with control media or NA $10 \mu M$ for 1, 2, 6 or 24 hours. RNA was isolated and mRNA levels of CCL6, CCL7, CCL12 and CXCL16 were determined by RT-PCR. Data are expressed as percentage of control values (set to $100 \%$ ). ${ }^{*} P<0.05$ versus control; ${ }^{*} P<0.01$ versus control; ${ }^{* * *} P<0.001$ versus control. Data are means \pm SE of $n=8$ replicates per group. (B) Astrocytes were incubated with control media (white columns), LPS $0.1 \mu \mathrm{g} / \mathrm{ml}$ (black columns) or LPS and NA $10 \mu \mathrm{M}$ (gray columns) for 1, 2, 6 or 24 hours. RNA was isolated and mRNA levels of CCL6, CCL7, CCL12 and CXCL16 were determined by RT-PCR. Data are expressed as percentage of control values (set to 100\%). ${ }^{*} P<0.05$ versus control; ${ }^{* *} P<0.01$ versus control; ${ }^{* * *} P<0.001$ versus control; ${ }^{\oplus} P<0.05$ versus $L P S ;{ }^{\oplus \Phi} P<0.01$ versus LPS; ${ }^{\oplus \Phi} P<0.001$ versus LPS. Data are means \pm SE of $n=8$ replicates per group. C, control; CCL6, chemokine (C-C motif) ligand 6; CCL7, chemokine (C-C motif) ligand 7; CCL12, chemokine (C-C motif) ligand 12; CXCL16, chemokine (C-X-C motif) ligand 16; LPS, lipopolysaccharide; NA, noradrenaline; RT-PCR; reverse transcription polymerase chain reaction; SE, standard error. 
RT-PCR measurements showed that while 6 hours of incubation with NA elevated CCL6 and CCL7 mRNA concentrations, the opposite effect happened for the other two chemokines analyzed (Figure 4A).

LPS caused an induction of CCL7 larger than the one caused by NA. It also induced CCL12 and CXCL16. For these three chemokines, NA showed an inhibitory effect in the presence of LPS similar to the one observed for CCL2 and CX3CL1 (Figure 4B).

In order to further analyze our results, ELISA studies were performed for these four chemokines; however, detectable amounts were only found for CCL6. LPS treatment caused an increase in the accumulation of CCL6 that was prevented by NA, while no modifications were caused by NA alone (Figure 5 ).

\section{Dual effect of NA on pro-inflammatory mediators}

According to our hypothesis, NA may participate in regulating the levels of certain mediators used by brain cells to communicate, maintaining constitutive production in basal conditions. However, when an exaggerated production of some of these mediators is induced as a result of an injury, NA effect is reversed helping to maintain homeostasis. Based on this, we analyzed NA effect on the expression of some well-known pro-inflammatory mediators, namely, inflammatory cytokines. Due to the large number of existing pro-inflammatory cytokines, a selection was made as in the case of the chemokines. Interleukin-1 beta (IL-1 $\beta$ ) and TNF $\alpha$ were selected, since these cytokines are known to be produced by astrocytes, and have toxic and trophic actions on neurons [24-26]. As

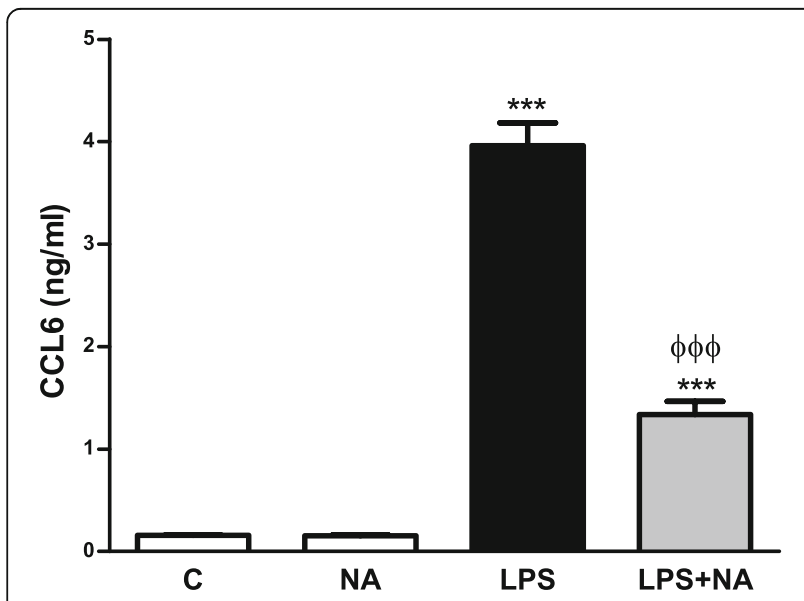

Figure 5 In the presence of LPS NA inhibits CCL6 production by astrocytes. Astrocytes were incubated with control media, NA $10 \mu \mathrm{M}$ or LPS $0.1 \mu \mathrm{g} / \mathrm{ml}$ alone or in combination with NA $10 \mu \mathrm{M}$ for 24 hours. CCL6 levels in the media were assessed by ELISA. ${ }^{* *} P$ $<0.001$ versus control; ${ }^{\circledR \Phi \Phi} P<0.001$ versus LPS. Data are means \pm SE of $\mathrm{n}=12$ replicates per group. C, control; CCL6, chemokine (C-C motif) ligand 6; ELISA, enzyme-linked immunosorbent assay; LPS, lipopolysaccharide; NA, noradrenaline; SE, standard error. shown in Figure 6, IL-1 $\beta$ mRNA levels were elevated as a result of the incubation of astrocytes with NA, reaching their maximal after 2 hours and decreasing afterwards to control levels. On the other hand, TNF $\alpha$ expression was reduced by NA. However, in the presence of LPS, NA effect was inhibitory for both cytokines (Figure 6B). This effect was also observed when measuring the concentration of TNF $\alpha$ released to the culture medium (Figure 6C).

Together with chemokines and cytokines, certain enzymes are key regulators of the inflammatory response. Nitric oxide synthase 2, inducible (NOS2) is known to be inhibited by NA, contributing to the antiinflammatory and neuroprotective actions of NA [20,27]. Another enzyme which catalyzes the production of multiple products, many of which have inflammatory potential, is cyclooxygenase-2 (COX-2). As shown in Figure 7, NA treatment elevated COX-2 mRNA and in the presence of LPS its effect was amplified (Figure 7B).

The pattern found for COX-2 production is different from those found for all the other proteins analyzed. This may be related to the differences in regulatory mechanisms. Based on the data, we decided to examine the effects of LPS and NA on COX-2 pathways further, particularly $\mathrm{PGE}_{2}$. To this end, $\mathrm{PGE}_{2}$ concentration in the culture media was measured by EIA and allowed us to observe modifications similar to those found for COX-2 (Figure 7C).

\section{Discussion}

The present study demonstrates the induction of CX3CL1 by NA in astrocytes. Given the neuroprotective actions described for CX3CL1 [28], its regulation by NA could help to explain the mechanisms through which NA protects neurons against different stimuli [29-31]. However, besides its anti-inflammatory and neuroprotective roles, CX3CL1, in its membrane anchored and soluble forms, also functions as a chemoattractant able to activate inflammatory cells [32]. In fact, an exaggerated response of CX3CL1 to certain injuries can lead to irreversible neuronal damage [16-18].

Like CX3CL1, CCL2 is another chemokine known to have certain protective actions in the CNS [33-37], but is also able to potentiate the inflammatory response and cause cell damage [38-40]. Similarly to CX3CL1, we had previously described CCL2 induction by NA in astrocyte cultures [9], as well as in mouse brain cortex astrocytes [41] and its complex regulation by different adrenergic receptors [42]. Both cytokines are expressed by cultured astrocytes and seem to be independently regulated by certain stimuli [43].

However, despite these dual effects of CCL2 and CX3CL1, NA is known to reduce brain inflammation $[5,44]$ and prevent the progression of pathologies with a neuroinflammatory component [45-48]. This led us to hypothesize that in basal conditions, NA may help to 

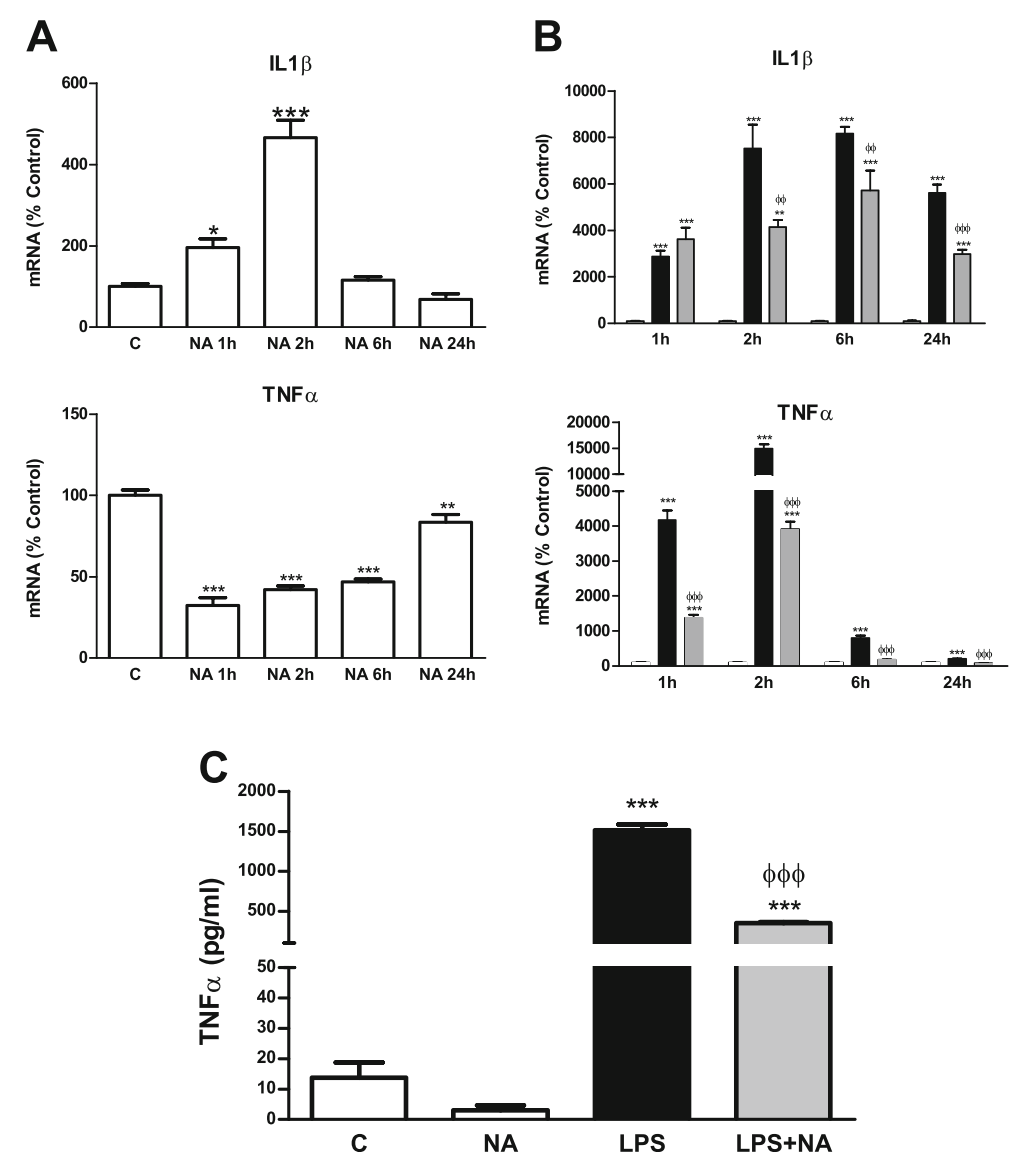

Figure 6 NA effects on different cytokines. (A) Astrocytes were incubated with control media or NA $10 \mu M$ for 1, 2, 6 or 24 hours. RNA was isolated and mRNA levels of IL-1 $\beta$, TNFa and IFNy were determined by RT-PCR. Data are expressed as percentage of control values (set to 100\%). ${ }^{*} P<0.05$ versus control; ${ }^{* *} P<0.01$ versus control; ${ }^{* * *} P<0.001$ versus control. Data are means \pm SE of $n=8$ replicates per group. (B) Astrocytes were incubated with control media (white columns), LPS $0.1 \mu \mathrm{g} / \mathrm{ml}$ (black columns) or LPS and NA $10 \mu \mathrm{M}$ (gray columns) for 1, 2, 6 or 24 hours. RNA was isolated and mRNA levels of IL-1 $\beta$, TNFa and IFNY were determined by RT-PCR. Data are expressed as percentage of control values (set to $100 \%)$. ${ }^{* *} P<0.01$ versus control; ${ }^{* * *} P<0.001$ versus control; ${ }^{\oplus \Phi} P<0.01$ versus $L P S ; ~{ }^{\oplus \Phi} P<0.001$ versus LPS. Data are means $\pm S E$ of $n=8$ replicates per group. (C) Astrocytes were incubated with control media, NA $10 \mu \mathrm{M}$, LPS $0.1 \mu \mathrm{g} / \mathrm{ml}$ alone or in combination with NA $10 \mu \mathrm{M}$ for 24 hours. TNFa levels in the media were assessed by ELISA. ${ }^{* * *} P<0.001$ versus control, ${ }^{\Phi \Phi \Phi} P<0.001$ versus LPS. Data are means $\pm S E$ of $n=12$ replicates per group. C, control; ELISA, enzyme-linked immunosorbent assay; IFNy, interferon gamma; IL-1 $\beta$, interleukin-1 beta; LPS, lipopolysaccharide; NA, noradrenaline; RT-PCR, reverse transcription polymerase chain reaction; SE, standard error; TNFa, tumor necrosis factor alpha.

maintain CNS levels of different cytokines and chemokines necessary for homeostasis, but under inflammatory conditions, NA prevents an exaggerated production of some of these mediators with neurotoxic potential. In agreement with this, the maximal concentration of CX3CL1 production reached after NA treatment was considerably lower than that observed when LPS was used instead.

Based on our data, the reduction of brain NA levels observed in certain neurodegenerative pathologies, such as Alzheimer's [6] and Parkinson's [49] diseases, could be the reason for the parallel loss of the constitutive production of these chemokines. In fact, there is a decreased production of CX3CL1 in the cortex and hippocampus of transgenic amyloid precursor protein
(APP) mice together with an elevated accumulation of amyloid beta $(A \beta)[50]$. This could be in agreement with those studies where the reduction of NA production either by depletion of locus coeruleus neurons [51] or by genetic alterations [52] aggravated the neurological damage in models of Alzheimer's disease.

While NA effects on chemokines production seem to be mediated through its interaction with adrenergic receptors $[9,42]$, the presence of major histocompatibility complex class II molecules or cluster of differentiation 14 (CD14) proteins detected on the surface of stimulated astrocytes [53,54], suggests that the promoter requirements and pathways leading to the production of the different chemokines and cytokines analyzed may be different for both types of stimuli. 


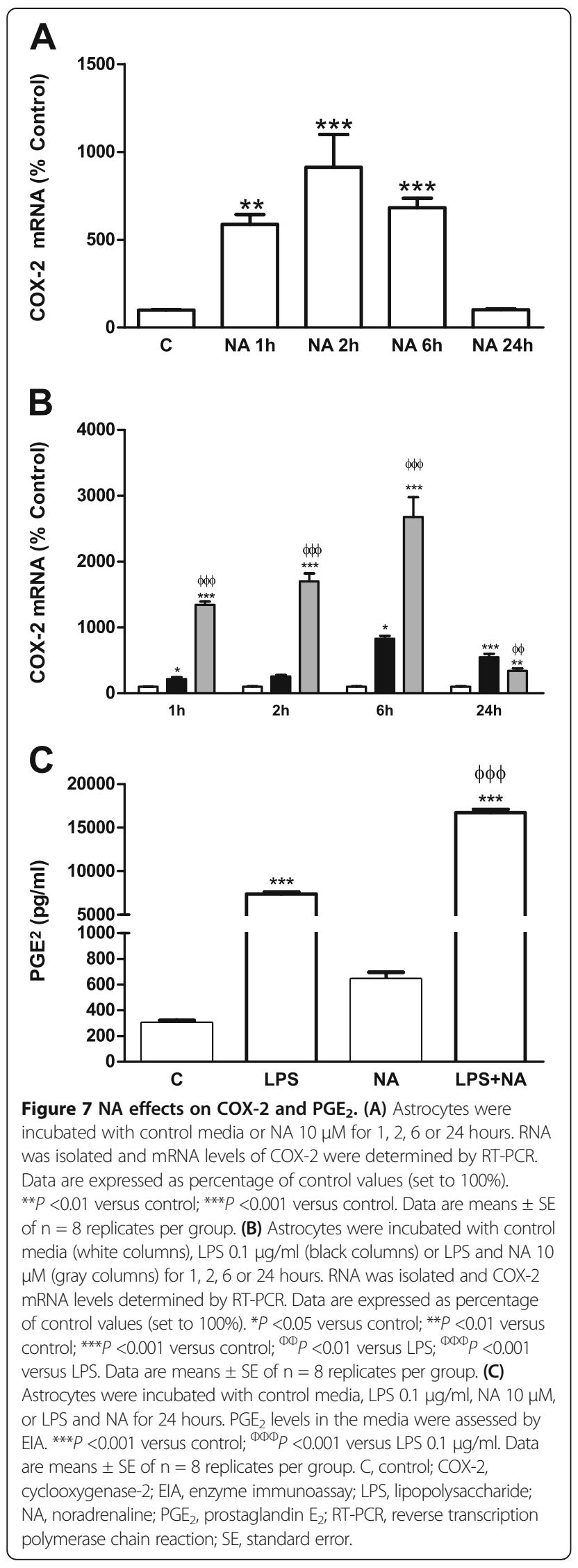

The PCR analysis of CCL7, CCL12 and CXCL16 reveals an inhibitory effect of NA in the presence of LPS for all of them, independently of NA effect in the absence of other stimulus. A similar pattern is observed for CCL6 concentration in the culture medium, while its mRNA levels are reduced by LPS. This indicates that in this case some post-transcriptional alterations are involved, resulting in a regulation similar to that observed for the other cytokines and chemokines analyzed.

Our results suggest that in the presence of an inflammatory stimulus, such as LPS, the actions of NA with respect to the expression of certain cytokines seem to be oriented towards the reversion of LPS alterations, independently of the changes NA may produce in the absence of other stimuli. This possibility constitutes an interesting new research subject, since the modification of NA actions on astrocytes are probably due to transformations caused by the activation of these cells as a response to an injury or any threat to homeostasis [55].

The induction by LPS of IL- $1 \beta$ and TNF $\alpha$ is a wellknown response of astrocytes [56,57], and the inhibition caused by NA is in agreement with its neuroprotective effects due to the pro-inflammatory nature of these cytokines [58,59]. On the other hand, it was more surprising to detect the induction of IL- $1 \beta$ by NA. Nevertheless, this could be in agreement with the above mentioned hypothesis, since this cytokine has been described to help protect neurons against certain types of injuries [24,26].

NA effects on COX-2 production have been previously analyzed in microglia by Schlachetzki et al. [60]. They also observed an induction by NA that was potentiated by LPS. Similarly to what we found for astrocytes, their work also describes the release of $\mathrm{PGE}_{2}$ by microglia in response to LPS or NA and the boosting of this effect by the combination of both treatments. Considering the involvement of $\mathrm{PGE}_{2}$ in the development of neuroinflammation [61], this effect of NA seems contradictory with its neuroprotective actions. However, $\mathrm{PGE}_{2}$ is another mediator for whom neuroprotective actions have also been discovered [62-65]. While this could help to explain our results, the additive actions of LPS and NA on COX-2 and $\mathrm{PGE}_{2}$ expression, eliminate the possibility of a simplistic explanation according to which NA reverses the changes caused by an inflammatory stimulus on astrocytes. This confirms the complex nature of NA mechanisms of action and, in particular, reveals the need to study $\mathrm{PGE}_{2}$ interactions with neurons and if the presence of NA also modulates the response to $\mathrm{PGE}_{2}$.

\section{Conclusions}

While NA neuroprotective actions are largely confirmed by different studies, its mechanisms of action are not well-known. MCP-1 and CX3CL1 induction could help to explain some of NA effects due to their ability to 
prevent neuronal damage under diverse conditions. However, such effect could be reversed when these and other mediators are produced in an exaggerated/uncontrolled manner. The data presented here indicate that NA may help to maintain the production of certain chemokines, while preventing their overproduction and subsequent toxicity. Further investigations of in vivo models may help confirm this hypothesis, extend the understanding of NA neuroprotective role and hopefully facilitate the development of NA-based therapies for neurodegenerative diseases.

\section{Abbreviations}

ANOVA: Analysis of variance; APP: Amyloid precursor protein; A 3 : Amyloid beta; CCL12: Chemokine (C-C motif) ligand 12; CCL2: Chemokine (C-C motif) ligand 2; CCL6: Chemokine (C-C motif) ligand 6; CCL7: Chemokine (C-C motif) ligand 7; CCR2: C-C chemokine receptor type 2; CD14: Cluster of differentiation 14; CNS: Central nervous system; COX-2: Cyclooxygenase-2; CX3CL1: Chemokine (C-X3-C motif) ligand 1; CXCL16: Chemokine (C-X-C motiff ligand 16; DMEM: Dulbecco's modified Eagle's medium; EIA: Enzyme immunoassay; ELISA: Enzyme-linked immunosorbent assay; EU: Endotoxin units; GAPDH: Glyceraldehyde 3-phosphate dehydrogenase; GFAP: Glial fibrillary acidic protein; IFNY: Interferon gamma; IL-1 1 : Interleukin-1 beta; LPS: Lipopolysaccharide; MCP-1: Monocyte chemotactic protein-1; MCP3: Monocyte chemotactic protein-3; MCP-5: Monocyte chemotactic protein-5; NA: Noradrenaline; NOS2: Nitric oxide synthase 2 inducible; PCR: Polymerase chain reaction; $\mathrm{PGE}_{2}$ : Prostaglandin $\mathrm{E}_{2} ;$ GPCR: Real-time polymerase chain reaction; qRT-PCR: Real-time reverse transcription polymerase chain reaction; RT-PCR: Reverse transcription polymerase chain reaction; SCYB16: Small inducible cytokine subfamily B member 16; SE: Standard error; TNFa: Tumor necrosis factor alpha.

\section{Competing interests}

The authors declare that they have no competing interests.

\section{Authors' contributions}

AH carried out the cell assays, RT-PCR, data acquisition and helped to draft the manuscript. JC, BG and JL contributed to analysis and interpretation of data, and drafted and critically revised the manuscript. JM performed cell cultures and treatments, conceived the study, participated in its design and coordination, and helped to draft the manuscript. All authors read and approved the final manuscript.

\section{Acknowledgements}

This work was supported by a grant from the Spanish Ministry of Science and Innovation (SAF2010-21948) to JM. AH is a fellow of Centro de Investigación Biomédica en Red de Salud Mental (CIBERSAM), Madrid, Spain. $J C$ and BB are (Juan de la Cierva) and (Ramón y Cajal) fellows (Ministerio de Economía y Competitividad), respectively.

\section{Author details}

'Departamento de Farmacología, Facultad de Medicina, Universidad Complutense de Madrid (UCM), Centro de Investigación Biomédica en Red de Salud Mental (CIBERSAM), Instituto de Investigación Hospital 12 de Octubre (Imas12), Instituto de Investigación en Neuroquímica UCM, Avenida Complutense s/n, Madrid 28040, Spain. ${ }^{2}$ Departamento de Psiquiatría, Facultad de Medicina, Universidad Complutense de Madrid (UCM), Centro de Investigación Biomédica en Red de Salud Mental (CIBERSAM), Instituto de Investigación Hospital 12 de Octubre (Imas12), Instituto de Investigación en Neuroquímica UCM, Avenida Complutense s/n, Madrid 28040, Spain.

Received: 7 May 2013 Accepted: 29 June 2013

Published: 9 July 2013

\section{References}

1. Brunello N, Blier P, Judd LL, Mendlewicz J, Nelson CJ, Souery D, Zohar J, Racagni G: Noradrenaline in mood and anxiety disorders: basic and clinical studies. Int Clin Psychopharmacol 2003, 18:191-202.
2. McIntyre CK, McGaugh JL, Williams CL: Interacting brain systems modulate memory consolidation. Neurosci Biobehav Rev 2012, 36:1750-1762.

3. Morilak DA, Barrera G, Echevarria DJ, Garcia AS, Hernandez A, Ma S, Petre CO: Role of brain norepinephrine in the behavioral response to stress. Prog Neuropsychopharmacol Biol Psychiatry 2005, 29:1214-1224.

4. O'Donnell J, Zeppenfeld D, McConnell E, Pena S, Nedergaard M: Norepinephrine: a neuromodulator that boosts the function of multiple cell types to optimize CNS performance. Neurochem Res 2012, 37:2496-2512.

5. Feinstein DL, Heneka MT, Gavrilyuk V, Dello RC, Weinberg G, Galea E: Noradrenergic regulation of inflammatory gene expression in brain. Neurochem Int 2002, 41:357-365.

6. Weinshenker D: Functional consequences of locus coeruleus degeneration in Alzheimer's disease. Curr Alzheimer Res 2008, 5:342-345.

7. Marien MR, Colpaert FC, Rosenquist AC: Noradrenergic mechanisms in neurodegenerative diseases: a theory. Brain Res Brain Res Rev 2004, 45:38-78.

8. Gee JR, Keller JN: Astrocytes: regulation of brain homeostasis via apolipoprotein E. Int J Biochem Cell Biol 2005, 37:1145-1150.

9. Madrigal JL, Leza JC, Polak P, Kalinin S, Feinstein DL: Astrocyte-derived MCP-1 mediates neuroprotective effects of noradrenaline. J Neurosci 2009, 29:263-267.

10. Conductier G, Blondeau N, Guyon A, Nahon JL, Rovere C: The role of monocyte chemoattractant protein MCP1/CCL2 in neuroinflammatory diseases. J Neuroimmunol 2010, 224:93-100.

11. Semple BD, Kossmann T, Morganti-Kossmann MC: Role of chemokines in CNS health and pathology: a focus on the CCL2/CCR2 and CXCL8/CXCR2 networks. J Cereb Blood Flow Metab 2010, 30:459-473.

12. Ono SJ, Nakamura T, Miyazaki D, Ohbayashi M, Dawson M, Toda M: Chemokines: roles in leukocyte development, trafficking, and effector function. J Allergy Clin Immunol 2003, 111:1185-1199.

13. Mehrad B, Keane MP, Strieter RM: Chemokines as mediators of angiogenesis. Thromb Haemost 2007, 97:755-762.

14. Belmadani A, Tran PB, Ren D, Miller RJ: Chemokines regulate the migration of neural progenitors to sites of neuroinflammation. J Neurosci 2006, 26:3182-3191.

15. Meucci O, Fatatis A, Simen AA, Miller RJ: Expression of CX3CR1 chemokine receptors on neurons and their role in neuronal survival. Proc Natl Acad Sci U S A 2000, 97:8075-8080.

16. Denes A, Ferenczi S, HalasZ J, Kornyei Z, Kovacs KJ: Role of CX3CR1 (fractalkine receptor) in brain damage and inflammation induced by focal cerebral ischemia in mouse. J Cereb Blood Flow Metab 2008, 28:1707-1721.

17. Fuhrmann M, Bittner $T$, Jung $C K$, Burgold $S$, Page RM, Mitteregger $G$, Haass C, LaFerla FM, Kretzschmar H, Herms J: Microglial Cx3cr1 knockout prevents neuron loss in a mouse model of Alzheimer's disease. Nat Neurosci 2010, 13:411-413.

18. Lee $\mathrm{S}$, Varvel NH, Konerth ME, Xu G, Cardona AE, Ransohoff RM, Lamb BT: CX3CR1 deficiency alters microglial activation and reduces beta-amyloid deposition in two Alzheimer's disease mouse models. Am J Pathol 2010, 177:2549-2562.

19. Desforges NM, Hebron ML, Algarzae NK, Lonskaya I, Moussa CE: Fractalkine mediates communication between pathogenic proteins and microglia: implications of anti-inflammatory treatments in different stages of neurodegenerative diseases. Int J Alzheimers Dis 2012, 201(2):345472.

20. Madrigal JL, Dello RC, Gavrilyuk V, Feinstein DL: Effects of noradrenaline on neuronal NOS2 expression and viability. Antioxid Redox Signal 2006, 8:885-892.

21. Cerbai F, Lana D, Nosi D, Petkova-Kirova P, Zecchi S, Brothers HM, Wenk GL, Giovannini MG: The neuron-astrocyte-microglia triad in normal brain ageing and in a model of neuroinflammation in the rat hippocampus. PLOS One 2012, 7:e45250.

22. Zlotnik A, Yoshie O: Chemokines: a new classification system and their role in immunity. Immunity 2000, 12:121-127.

23. Rosito M, Deflorio C, Limatola C, Trettel F: CXCL16 orchestrates adenosine A3 receptor and $\mathrm{MCP}-1 / \mathrm{CCL} 2$ activity to protect neurons from excitotoxic cell death in the CNS. J Neurosci 2012, 32:3154-3163.

24. Carlson NG, Wieggel WA, Chen J, Bacchi A, Rogers SW, Gahring LC: Inflammatory cytokines IL-1 alpha, IL-1 beta, IL-6, and TNF-alpha impart neuroprotection to an excitotoxin through distinct pathways. I Immunol 1999, 163:3963-3968.

25. Figiel I: Pro-inflammatory cytokine TNF-alpha as a neuroprotective agent in the brain. Acta Neurobiol Exp (Wars) 2008, 68:526-534. 
26. Shaftel SS, Griffin WS, O'Banion MK: The role of interleukin-1 in neuroinflammation and Alzheimer disease: an evolving perspective. J Neuroinflammation 2008, 5:7.

27. Dello RC, Boullerne Al, Gavrilyuk V, Feinstein DL: Inhibition of microglial inflammatory responses by norepinephrine: effects on nitric oxide and interleukin-1 beta production. J Neuroinflammation 2004, 1:9.

28. Re DB, Przedborski S: Fractalkine: moving from chemotaxis to neuroprotection. Nat Neurosci 2006, 9:859-861.

29. Kalinin S, Polak PE, Lin SX, Sakharkar AJ, Pandey SC, Feinstein DL: The noradrenaline precursor L-DOPS reduces pathology in a mouse model of Alzheimer's disease. Neurobiol Aging 2012, 33:1651-1663.

30. Traver S, Salthun-Lassalle B, Marien M, Hirsch EC, Colpaert F, Michel PP: The neurotransmitter noradrenaline rescues septal cholinergic neurons in culture from degeneration caused by low-level oxidative stress. $\mathrm{Mol}$ Pharmacol 2005, 67:1882-1891.

31. Madrigal JL, Feinstein DL, Dello RC: Norepinephrine protects cortical neurons against microglial-induced cell death. J Neurosci Res 2005, 81:390-396

32. Bazan JF, Bacon KB, Hardiman G, Wang W, Soo K, Rossi D, Greaves DR, Zlotnik A, Schall TJ: A new class of membrane-bound chemokine with a CX3C motif. Nature 1997, 385:640-644.

33. Bruno V, Copani A, Besong G, Scoto G, Nicoletti F: Neuroprotective activity of chemokines against N-methyl-D-aspartate or beta-amyloid-induced toxicity in culture. Eur J Pharmacol 2000, 399:117-121.

34. Elhofy A, Wang J, Tani M, Fife BT, Kennedy KJ, Bennett J, Huang D, Ransohoff RM, Karpus WJ: Transgenic expression of CCL2 in the central nervous system prevents experimental autoimmune encephalomyelitis. J Leukoc Biol 2005, 77:229-237.

35. Eugenin EA, D'Aversa TG, Lopez L, Calderon TM, Berman JW: MCP-1 (CCL2) protects human neurons and astrocytes from NMDA or HIV-tat-induced apoptosis. J Neurochem 2003, 85:1299-1311.

36. Godefroy D, Gosselin RD, Yasutake A, Fujimura M, Combadiere C, MauryBrachet R, Laclau M, Rakwal R, Melik-Parsadaniantz S, Bourdineaud JP, Rostène $\mathrm{W}$ : The chemokine CCL2 protects against methylmercury neurotoxicity. Toxicol Sci 2012, 125:209-218.

37. Zisman DA, Kunkel SL, Strieter RM, Tsai WC, Bucknell K, Wilkowski J, Standiford TJ: MCP-1 protects mice in lethal endotoxemia. J Clin Invest 1997, 99:2832-2836.

38. Kiyota T, Gendelman HE, Weir RA, Higgins EE, Zhang G, Jain M: CCL2 affects beta-amyloidosis and progressive neurocognitive dysfunction in a mouse model of Alzheimer's disease. Neurobiol Aging 2013, 34:1060-1068.

39. Ge S, Shrestha B, Paul D, Keating C, Cone R, Guglielmotti A, Pachter JS: The CCL2 synthesis inhibitor bindarit targets cells of the neurovascular unit, and suppresses experimental autoimmune encephalomyelitis. J Neuroinflammation 2012, 9:171.

40. Yang G, Meng Y, Li W, Yong Y, Fan Z, Ding H, Wei Y, Luo J, Ke ZJ: Neuronal MCP-1 mediates microglia recruitment and neurodegeneration induced by the mild impairment of oxidative metabolism. Brain Pathol 2011, 21:279-297.

41. Madrigal $J L$, Garcia-Bueno B, Hinojosa AE, Polak P, Feinstein DL, Leza JC: Regulation of MCP-1 production in brain by stress and noradrenalinemodulating drugs. J Neurochem 2010, 113:543-551.

42. Hinojosa AE, Garcia-Bueno B, Leza JC, Madrigal JL: Regulation of CCL2/MCP-1 production in astrocytes by desipramine and atomoxetine: involvement of alpha2 adrenergic receptors. Brain Res Bull 2011, 86:326-333.

43. Koyama Y, Kotani M, Sawamura T, Kuribayashi M, Konishi R, Michinaga S: Different actions of endothelin-1 on chemokine production in rat cultured astrocytes: reduction of CX3CL1/fractalkine and an increase in CCL2/MCP-1 and CXCL1/CINC-1. J Neuroinflammation 2013, 10:51.

44. O'Sullivan JB, Ryan KM, Curtin NM, Harkin A, Connor TJ: Noradrenaline reuptake inhibitors limit neuroinflammation in rat cortex following a systemic inflammatory challenge: implications for depression and neurodegeneration. Int J Neuropsychopharmacol 2009, 12:687-699.

45. Delaville C, Deurwaerdere PD, Benazzouz A: Noradrenaline and Parkinson's disease. Front Syst Neurosci 2011, 5:31.

46. Gustafson I, Westerberg E, Wieloch T: Protection against ischemia-induced neuronal damage by the alpha 2-adrenoceptor antagonist idazoxan: influence of time of administration and possible mechanisms of action. J Cereb Blood Flow Metab 1990, 10:885-894.

47. Polak PE, Kalinin S, Feinstein DL: Locus coeruleus damage and noradrenaline reductions in multiple sclerosis and experimental autoimmune encephalomyelitis. Brain 2011, 134:665-677.
48. Heneka MT, Ramanathan M, Jacobs AH, Dumitrescu-Ozimek L, Bilkei-Gorzo A, Debeir T, Sastre M, Galldiks N, Zimmer A, Hoehn M, Heiss WD, Klockgether T, Staufenbiel M: Locus ceruleus degeneration promotes Alzheimer pathogenesis in amyloid precursor protein 23 transgenic mice. J Neurosci 2006, 26:1343-1354.

49. Fornai F, di Poggio AB, Pellegrini A, Ruggieri S, Paparelli A: Noradrenaline in Parkinson's disease: from disease progression to current therapeutics. Curr Med Chem 2007, 14:2330-2334.

50. Duan RS, Yang X, Chen ZG, Lu MO, Morris C, Winblad B, Zhu J: Decreased fractalkine and increased IP-10 expression in aged brain of APP(swe) transgenic mice. Neurochem Res 2008, 33:1085-1089.

51. Kalinin S, Gavrilyuk V, Polak PE, Vasser R, Zhao J, Heneka MT, Feinstein DL: Noradrenaline deficiency in brain increases beta-amyloid plaque burden in an animal model of Alzheimer's disease. Neurobiol Aging 2007, 28:1206-1214

52. Hammerschmidt T, Kummer MP, Terwel D, Martinez A, Gorji A, Pape HC, Rommelfanger KS, Schroeder JP, Stoll M, Schultze J, Weinshenker D, Heneka MT: Selective loss of noradrenaline exacerbates early cognitive dysfunction and synaptic deficits in APP/PS1 mice. Biol Psychiatry 2013, 73:454-463.

53. Galea E, Reis DJ, Fox ES, Xu H, Feinstein DL: CD14 mediate endotoxin induction of nitric oxide synthase in cultured brain glial cells. J Neuroimmunol 1996, 64:19-28.

54. Guillemin G, Boussin FD, Croitoru J, Franck-Duchenne M, Le GR, Lazarini F, Dormont D: Obtention and characterization of primary astrocyte and microglial cultures from adult monkey brains. J Neurosci Res 1997, 49:576-591.

55. Pekny M, Nilsson M: Astrocyte activation and reactive gliosis. Glia 2005 , 50:427-434

56. Chung IY, Benveniste EN: Tumor necrosis factor-alpha production by astrocytes. Induction by lipopolysaccharide, IFN-gamma, and IL-1 beta. J Immunol 1990, 144:2999-3007.

57. Soliman ML, Combs CK, Rosenberger TA: Modulation of inflammatory cytokines and mitogen-activated protein kinases by acetate in primary astrocytes. J Neuroimmune Pharmacol 2013, 8:287-300.

58. Basu A, Krady JK, Levison SW: Interleukin-1: a master regulator of neuroinflammation. J Neurosci Res 2004, 78:151-156.

59. Montgomery SL, Bowers WJ: Tumor necrosis factor-alpha and the roles it plays in homeostatic and degenerative processes within the central nervous system. J Neuroimmune Pharmacol 2012, 7:42-59.

60. Schlachetzki JC, Fiebich BL, Haake E, de Oliveira AC, Candelario-Jalil E, Heneka MT, Hüll M: Norepinephrine enhances the LPS-induced expression of COX-2 and secretion of PGE2 in primary rat microglia. J Neuroinflammation 2010, 7:2.

61. Shi J, Wang Q, Johansson JU, Liang X, Woodling NS, Priyam P, Loui TM, Merchant M, Breyer RM, Montine TJ, Andreasson K: Inflammatory prostaglandin E2 signaling in a mouse model of Alzheimer disease. Ann Neurol 2012, 72:788-798.

62. Thery C, Dobbertin A, Mallat M: Downregulation of in vitro neurotoxicity of brain macrophages by prostaglandin E2 and a beta-adrenergic agonist. Glia 1994, 11:383-386.

63. Kim EJ, Kwon KJ, Park JY, Lee SH, Moon CH, Baik EJ: Neuroprotective effects of prostaglandin E2 or CAMP against microglial and neuronal free radical mediated toxicity associated with inflammation. J Neurosci Res 2002, 70:97-107.

64. Akaike A, Kaneko S, Tamura Y, Nakata N, Shiomi H, Ushikubi F, Narumiya S: Prostaglandin E2 protects cultured cortical neurons against N-methyl-Daspartate receptor-mediated glutamate cytotoxicity. Brain Res 1994, 663:237-243.

65. Cazevieille C, Muller A, Meynier F, Dutrait N, Bonne C: Protection by prostaglandins from glutamate toxicity in cortical neurons. Neurochem Int 1994, 24:395-398.

doi:10.1186/1742-2094-10-81

Cite this article as: Hinojosa et al:: Dual effects of noradrenaline on astroglial production of chemokines and pro-inflammatory mediators. Journal of Neuroinflammation 2013 10:81. 